\title{
Developmental Delay
}

National Cancer Institute

\section{Source}

National Cancer Institute. Developmental Delay. NCI Thesaurus. Code C116942.

Failure to meet, or late achievement of developmental milestones. 